\title{
Improving the accuracy of walking piezo motors
}

M. den Heijer, V. Fokkema, A. Saedi, P. Schakel, and M. J. Rost

Citation: Review of Scientific Instruments 85, 055007 (2014); doi: 10.1063/1.4878624

View online: http://dx.doi.org/10.1063/1.4878624

View Table of Contents: http://aip.scitation.org/toc/rsi/85/5

Published by the American Institute of Physics

\section{Articles you may be interested in}

A simple, compact, and rigid piezoelectric step motor with large step size

Review of Scientific Instruments 80, 085104 (2009); 10.1063/1.3197381

A nanopositioner for scanning probe microscopy: The KoalaDrive

Review of Scientific Instruments 83, 023703 (2012); 10.1063/1.3681444

Methods and instrumentation for piezoelectric motors

Review of Scientific Instruments 83, 033706 (2012); 10.1063/1.3694972

How are the behaviors of piezoelectric inertial sliders interpreted?

Review of Scientific Instruments 83, 093701 (2012); 10.1063/1.4748275

Ultra compact multitip scanning tunneling microscope with a diameter of $50 \mathrm{~mm}$

Review of Scientific Instruments 83, 033707 (2012); 10.1063/1.3694990

A piezo motor based on a new principle with high output force, rigidity and integrity: The Tuna Drive Review of Scientific Instruments 83, 115111 (2012); 10.1063/1.4767246

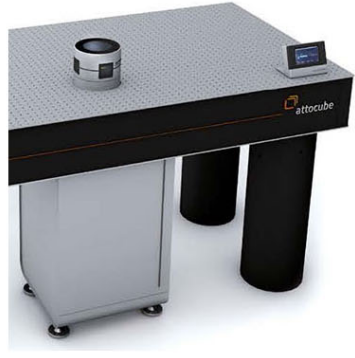

Obstruction free access

optical table with integrated cryocooler

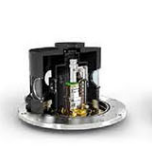

Various Objective 0ptions

\section{attoDRY800}

- Cryogenic Temperatures

- Ultra-Low Vibration

- Optical Table Included

- Fast Cooldown
$5 \%$ DISCOUNT

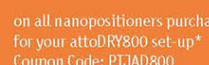

attocube

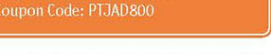




\title{
Improving the accuracy of walking piezo motors
}

\author{
M. den Heijer, V. Fokkema, A. Saedi, P. Schakel, and M. J. Rost ${ }^{\text {a) }}$ \\ Kamerlingh Onnes Laboratory, Leiden University, Niels Bohrweg 2, 2333 CA Leiden, The Netherlands
}

(Received 15 March 2013; accepted 4 May 2014; published online 27 May 2014)

\begin{abstract}
Many application areas require ultraprecise, stiff, and compact actuator systems with a high positioning resolution in combination with a large range as well as a high holding and pushing force. One promising solution to meet these conflicting requirements is a walking piezo motor that works with two pairs of piezo elements such that the movement is taken over by one pair, once the other pair reaches its maximum travel distance. A resolution in the pm-range can be achieved, if operating the motor within the travel range of one piezo pair. However, applying the typical walking drive signals, we measure jumps in the displacement up to $2.4 \mu \mathrm{m}$, when the movement is given over from one piezo pair to the other. We analyze the reason for these large jumps and propose improved drive signals. The implementation of our new drive signals reduces the jumps to less than $42 \mathrm{~nm}$ and makes the motor ideally suitable to operate as a coarse approach motor in an ultra-high vacuum scanning tunneling microscope. The rigidity of the motor is reflected in its high pushing force of $6.4 \mathrm{~N}$. (C) 2014 AIP Publishing LLC. [http://dx.doi.org/10.1063/1.4878624]
\end{abstract}

\section{INTRODUCTION}

Due to the ongoing progress in many application areas, like nanotechnology, scanning probe microscopes (SPMs), ultra-high vacuum (UHV), low temperatures, semiconductor industry, precision optics alignment, and microbiological cell manipulation, there is a strong demand for ultraprecise, rigid, and compact actuator systems that offer a high positioning resolution in combination with a large range. The increasing requirements on the accuracy of these motors down to the nmrange, inherently involves stringent demands on the precise operation and, thereby, rises fundamental questions and problems in relation to nanoscale friction. ${ }^{1-3}$ In addition, often a motor is required that has a high holding and a high pushing/pulling force.

One of these applications is the use of such a motor as a coarse approach motor in an UHV-SPM. It is the task of the coarse approach motor to bring the tip into close vicinity to the sample (within the operating range of the scanning piezo), while avoiding overshoots and a resolution destroying tip crash into the sample. To ensure a safe approach, the step size of the approach motor should be $\sim 10$ times smaller than the height range of the scanning piezo: with a typical height range of $1 \mu \mathrm{m}$ the step size including possible overshoots should be less than $100 \mathrm{~nm}$. Several different piezoelectric motors are applied, like the walker introduced by Pan, ${ }^{4-9}$ the inchworm type ${ }^{10-21}$ the Besocke beetle type, ${ }^{22-31}$ and inertial sliders. ${ }^{32-38}$ However, despite some advantages all of these motors have some severe disadvantages.

The range of the "Pan"-walker and the inchworm can be mm's and is limited only by the length of the slider and the accuracy of its manufacturing. Both motors can deliver the highest holding and pushing force (in the order of $0.5 \mathrm{~N}$ ) of the motors discussed. However, a further increase of this force is hardly possible, as it is limited by the friction of the

a)Electronic mail: rost@physics.leidenuniv.nl piezo bearings. If the bearings are too loose, the motors do not work at all. If, on the other hand, the bearings are too tight, the motors get stuck. Another disadvantage of these motors is that their reliability in practical applications is low due to their complex structure, strict fabrication tolerances, and complex electronic control. The beetle, which works with a slip-stick principle on a circular ramp, is more reliable in this respect. However, the step size is determined statistically and the total range is significantly less than the "Pan"-walker or the inchworm. In addition, the beetle suffers from stability problems. ${ }^{30,31,39,40}$ Inertial sliders are relatively simple and, depending on their design, also reliable. However, the step size is determined statistically due to the slip-stick motion, the pushing force is limited, and they are usually not very rigid making them prone to vibrations. It is possible to increase the pushing force and the rigidity by pre-loading the slider with an additional spring, but this usually decreases the reliability. Other interesting new designs of linear motors with improved performance can be found in Refs. 41-44. However, all of these motors have disadvantages in comparison to the motor described in this paper: either the pushing force is significantly smaller and the rigidity therefore less, or the motor shows large jumps in the displacement, or the motor is not designed for operation in UHV.

Recent developments in video-rate SPM, see Refs. 45-48 and references therein, uniquely enable the real-time, in situ observation of dynamic and fast processes on an atomic scale, like graphene growth, ${ }^{49}$ thin (polycrystalline) film deposition, ${ }^{50}$ grain growth, and grain boundary migration, ${ }^{51}$ copper electrodeposition in the presence of additives, ${ }^{52}$ and real-time ion erosion..$^{53}$ As the coarse approach motor is part of the mechanical loop that connects the tip to the sample, the rigidity and mechanical stability of the motor is crucial for high-speed imaging, especially as the scanning line frequency can approach even several $\mathrm{kHz}$. If the line frequency, or a higher harmonics of it, hits a resonance frequency of the mechanical loop, imaging gets not only strongly distorted, but 
it also often leads to a tip crash. ${ }^{45-47}$ As the pushing and holding force of a motor is directly related to its rigidity, the higher these forces are the better this motor is suited for an application as coarse approach motor in a high-speed SPM.

In this paper, we investigate the performance of a walking piezo motor with application as a coarse approach motor in an UHV scanning tunneling microscope (STM). Using a scanning electron microscope (SEM), we directly measure the displacement of the motor on a nm scale. When applying the typical drive signals we find surprisingly large jumps in the displacement, up to $2.4 \mu \mathrm{m}$. We analyze the reason for these jumps and propose, based on our results, improved drive signals. Implementing our new drive signals, the size of the jumps is reduced to less than $42 \mathrm{~nm}$. This makes this motor ideally suited to work as a coarse approach motor in a highspeed UHV-SPM. It is expected that our new drive signals will improve the performance of every piezo motor that is working with a similar principle. Based on the final microscope, which operates now for more than 3 years, we provide further information on the reliability and performance of these motors and demonstrate that a resolution of $27 \mathrm{pm}$ can be achieved.

\section{THE WALKING PIEZO MOTOR}

The advantages of walking piezo motors in comparison to other piezo motors have been noticed both in research and in industry. ${ }^{54-58}$ The motors can be fabricated in different dimensions depending on the particular needs and their application. The motor used in this research has the following dimensions: $22 \times 19 \times 11 \mathrm{~mm}^{3}$. It has a pushing force of $6.4 \mathrm{~N}$ and a holding force of $7.3 \mathrm{~N}$, which is more than 10 times higher than the "Pan"-walker. It has a range of $80 \mathrm{~mm}$, which is limited only by the length of the sliding bar used. It can move with speeds up to $15 \mathrm{~mm} / \mathrm{s}$, it is vacuum compatible $\left(10^{-7}\right.$ Torr), and it has been tested to operate at temperatures up to $70{ }^{\circ} \mathrm{C}$. On the basis of the dimensions and the properties of the used piezo elements as well as the voltage range and the resolution of our drive electronics, one would expect that the step size is adjustable between $1 \mathrm{~nm}$ and $3 \mu \mathrm{m}$, and that a resolution better than $1 \mathrm{~nm}$ can be achieved in bending mode, i.e., operating the motor within the range of a piezo pair. We will show that it is even possible to control the motor with step sizes of only $27 \mathrm{pm}$.

Figure 1(a) shows a cut-open drawing of the motor: a roll bearing pushes, due to several leaf springs, a sliding ceramic bar against the piezo legs that are mounted in a housing. The motor is working with 4 piezo legs that can extend and shrink along the Z-direction as well as bend along the positive and negative $\mathrm{X}$-direction, and that are electrically coupled in two pairs, A and B, see Fig. 1(b). Each piezo leg consists of two individual piezos that are fused together, and that can extend and shrink separately, see zoom-in of Fig. 1(b). As the total displacement of one leg is very small with respect to its dimensions, the absolute displacement of the top of one leg can be approximated by a linear relation with respect to the applied the voltages, $V_{1}$ and $V_{2}$, to the electrodes

$$
\begin{aligned}
& x=k_{x}\left(V_{2}-V_{1}\right), \\
& z=k_{z}\left(V_{2}+V_{1}\right) .
\end{aligned}
$$
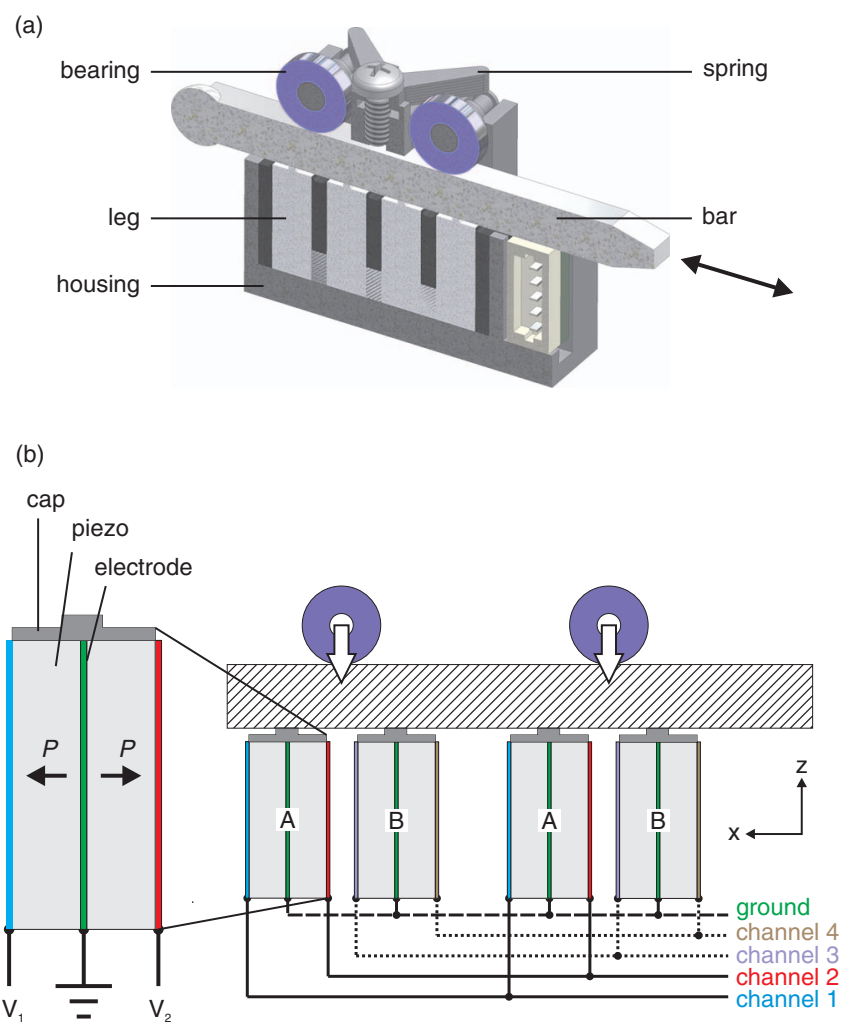

FIG. 1. The walking piezo motor. (a) Cut-open drawing of the motor: a roll bearing pushes, due to several leaf springs, a sliding ceramic bar against the piezo legs that are mounted in a housing. (b) The motor is working with two sets of piezo pairs, A and B, that both can extend and shrink along the $\mathrm{Z}$-direction as well as bend along the positive and negative $\mathrm{X}$-direction. $\mathrm{P}$ denotes the polarization direction of the piezos.

The sensitivities $k_{x}$ and $k_{z}$ depend on the piezoelectric material and very strongly also on the geometry of a leg. In the hypothetical case that $k_{x}=k_{z}$, the top of one (pair of) $\operatorname{leg}(\mathrm{s}$ ) could move anywhere inside a square outline as sketched in Fig. 2(a). On the basis of the dimensions of the piezo legs, we can derive the relation between the two sensitivities ${ }^{59}$

$$
k_{x} \times 0,548=k_{z} .
$$

Due to this relation, the range of a leg is decreased in the Z-direction by $\approx 45 \%$, which leads to a rhombic outline as shown in Fig. 2(b). We will determine the sensitivities from our measurements.

All further shown range -diagrams depict the real motion of the piezo legs in our experiments, which we carefully controlled by applying specific voltages to the four channels, (a)

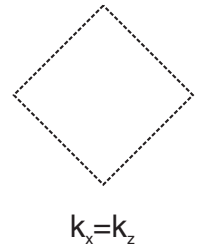

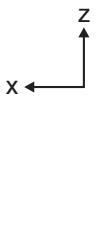

(b)

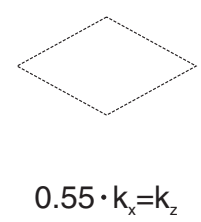

FIG. 2. X-and Z-range of the top of one piezo leg: (a) with equal sensitivities $\left(k_{x}=k_{z}\right)$ and (b) with the sensitivities according to the used motor $\left(k_{x} \times 0.548\right.$ $=k_{z}$ ). 
see Fig. 1(b). However, it is to mention that the real motion in $\mathrm{Z}$ is even further reduced due to the spring-loaded roll bearing.

There are many ways of moving the pairs that will result in a final displacement of the bar. There are two typical modes of operation applied for walking piezo motors, which we define as the walking mode and the fine mode. In the following description, we assume that left corresponds to the forward direction. Due to symmetry, the arguments hold equally well for the backwards direction.

In the walking mode, which is depicted in Fig. 3(a), the bar is pushed forward by moving pair A from the right to the left over the top edge of the rhombic outline (see red arrow). At the same time, pair B is returned to the starting position of pair A by moving it from left to right over the bottom edge of the rhombic outline (see blue arrow). The whole procedure can be repeated by interchanging the role of pair A and pair B. The drive signals in the walking mode are usually applied such that the motor makes always at least one full step. However, the step size can be varied typically between 0.3 and $3 \mu \mathrm{m}$ by limiting the drive signals, as indicated in Fig. 3(b).

The fine mode is depicted in Fig. 3(c) as a sequence of individual pair movements. First pair A slowly bends to the left pushing the bar forward (1). When pair A has reached the end of its range, pair B moves up and firmly clamps the bar (2). Pair A returns to its starting position (3), before pair B finally releases the bar (4). As the bar is held only by one pair during phase (1) operating in bending mode, the displacement of the bar can be easily made as slow as desired and it can be stopped or reversed in direction at any time. Therefore, the resolution is expected to be only limited by the resolution and the noise of the control voltage (neglecting piezo creep). The relation is about $1 \mathrm{~nm}$ for a voltage of $15 \mathrm{mV}$. The range of the bending phase (1) should be $3 \mu \mathrm{m}$ for our motor. Considering the displacement accuracy of the bar, especially on a nm scale, serious questions arise about possible positional fluctuations during the phases (2), (3), and (4), after the maximum range has been reached. We will address this point in Sec. III A-III C. (a)

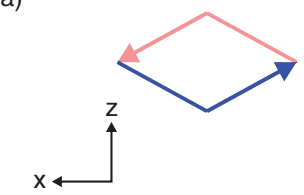

(b)

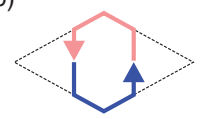

(c)

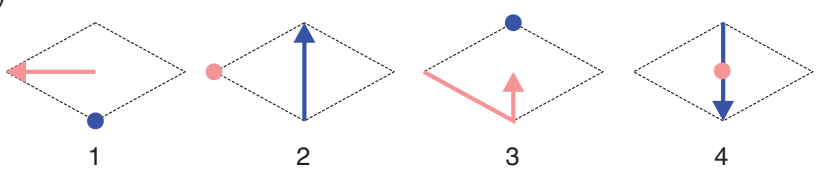

FIG. 3. Standard drive signals. Walking mode with full step size (a) and reduced step size (b). (c) Fine mode with the individual phases: bend (1), clamp (2), return (3), and release (4). Light red represents piezo pair A, blue represents pair B, and the black dashed lines indicate the rhombic outline, to which the movement of the legs is confined.

\section{RESULTS}

\section{A. Standard drive signal: Fine mode}

It is essential to use the fine mode when applying the motor as an approach motor in a STM, because the step size should be in the order of $100 \mathrm{~nm}$ (or less), as described in the Introduction. In addition, the motor should operate without (temporary) overshoots, sudden positional fluctuations, and creep. To quantify the behavior of the motor in fine mode in more detail, we placed the motor in a SEM. A small piece of grounded metal was attached to the end of the bar to prevent image distortions caused by electron beam charging of the bar. We view the metal on the bar from above, as shown in Fig. 4(d), and record a movie while the motor is running at two different speeds. Figures 4(a)-4(c) show three typical frames taken of such a movie.

A quantitative analysis of the frames not shown in this paper reveals that both between frames (a) and (b), and between frames (b) and (c), the bar moves slowly $(0.2 \mu \mathrm{m} / \mathrm{s}$ and $1.2 \mu \mathrm{m} / \mathrm{s}$, respectively) over a total distance of $1.7 \mu \mathrm{m}$. Therefore, the total X-range of the rhombic outline shown in Fig. 2(b) corresponds to $3.4 \mu \mathrm{m}$, whereas the total Z-range of the rhombic outline is only $\approx 1.9 \mu \mathrm{m}$ (neglecting the further decrease due to the spring-loaded ball bearing). This leads to the following sensitivities:

$$
\begin{aligned}
& k_{x}=68 \pm 5 \mathrm{~nm} / \mathrm{V}, \\
& k_{z}=37 \pm 3 \mathrm{~nm} / \mathrm{V} .
\end{aligned}
$$

The observed smooth movement for both speeds, between frames (a) and (b), and between frames (b) and (c), is very different from the movement captured by frames (b) and (c) that each show two positions of the bar. This is possible, because a complete SEM image is obtained by two sets of interlacing lines (first the odd and then the even lines are recorded). In these frames, the bar is displaced over $2.4 \mu \mathrm{m}$ within $0.05 \mathrm{~s}$. We shall refer to these fast movements as jumps in the rest of this text.

From the above analysis, we conclude that the range of bending is $1.7 \mu \mathrm{m}$. Strikingly, large jumps of typically 2.4 $\mu \mathrm{m}$ occur after the bending pair has reached its maximum stroke. Determined by the selected speed of our drive signals, these jumps occur in less than $0.05 \mathrm{~s}$ during the phases (2),

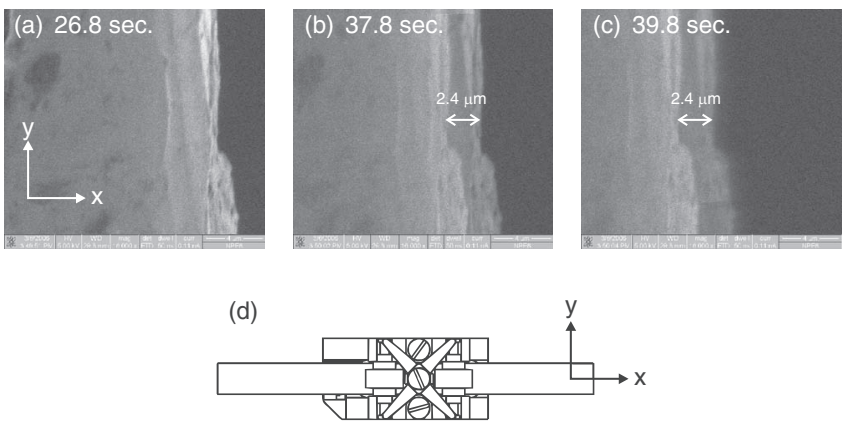

FIG. 4. SEM images during the movement: (a)-(c) three selected frames from a movie taken at the end of the bar with 20 images/s during its motion. The motor is controlled in fine mode. Please notice the jumps of $2.4 \mu \mathrm{m}$ within frame (b) and (c). (d) represents a schematic of the viewing direction. 
(3), and (4), see Fig. 3(c). With these large jumps, the motor is not suited for an application as coarse approach motor in a SPM.

Assuming a perfectly manufactured motor, proper drive signals, and piezo legs with equal dimensions, properties, and alignment, there is no obvious reason for such large jumps. One might speculate about the following possibility. The fine mode only works properly, if the phases 2 (clamp), 3 (return), and 4 (release), really do not move the bar in the X-direction. The very fast movement of these three phases (in less than $0.05 \mathrm{~s}$ ) requires a high accuracy on the individual timing of the applied voltages, see Fig. 1(b). Deviations might lead to the fact that the bar is dragged along the $\mathrm{X}$-direction during the return phase 3 (see Fig. 3(c)). Inertia might also play a role in this case, especially for high driving speeds. As an example, the bar might slip to the right (during phase 3), as long as pair B has not built up enough friction force to stop the movement. Another example might be the inertia of the bar between phases 1 and 2. Again, as long as pair B has not built up enough friction force, the bar might slip to the left.

Using the SEM, we also determined the resolution of the motor when operating it only within the maximum X-range of one piezo pair such that the movement does not have to be taken over by the other pair. Although the accuracy of this measurement was fully limited by the resolution of our high-resolution SEM, we could derive an accuracy/resolution of the motion that must be better than $1 \mathrm{~nm}$. This result is also confirmed by the Z-stability of our home-build STM (see Sec. III F), in which we applied the here described motors as coarse approach motors.

Based on the above described jumps and the considered possibilities for their existence, we further quantify the behavior of the motor with special test signals.

\section{B. Test drive signal: Z-displacement}

To allow the easy application of test signals, we developed a program (using LabView together with a National Instruments DAC: NI USB-6251 BNC) that enables us to apply any desired signal to each of the four channels of the motor: the output of the NI-DAC is amplified with piezo drivers.

Concerned about the very fast movement of the clamprelease-clamp phase in Sec. III A, the basic idea is to clearly separate the phases (2), (3), and (4) in time. In addition, we want to slow down the movement such that inertial effects can be ruled out. We applied the drive signals as depicted in Fig. 5(b) to the motor. First pair A moves up in pure $\mathrm{Z}$ direction within $2 \mathrm{~s}$ (phase 1), then it stays there for $2 \mathrm{~s}$ (phase 2), and finally moves back down in $2 \mathrm{~s}$ (phase 3 ), while pair B remains at the bottom during this entire period. To complete the characterization, we also interchanged the role of pair A and pair B, and, in addition, moved both pairs simultaneously. Applying these drive signals, an ideal motor should show no displacements along the $\mathrm{X}$-direction.

Similar as described above, we recorded a movie of the displacement of the bar using the SEM. We developed an image cross-correlation code in Matlab that processes the frames of the movie to obtain the numerical displacement data. The (a)
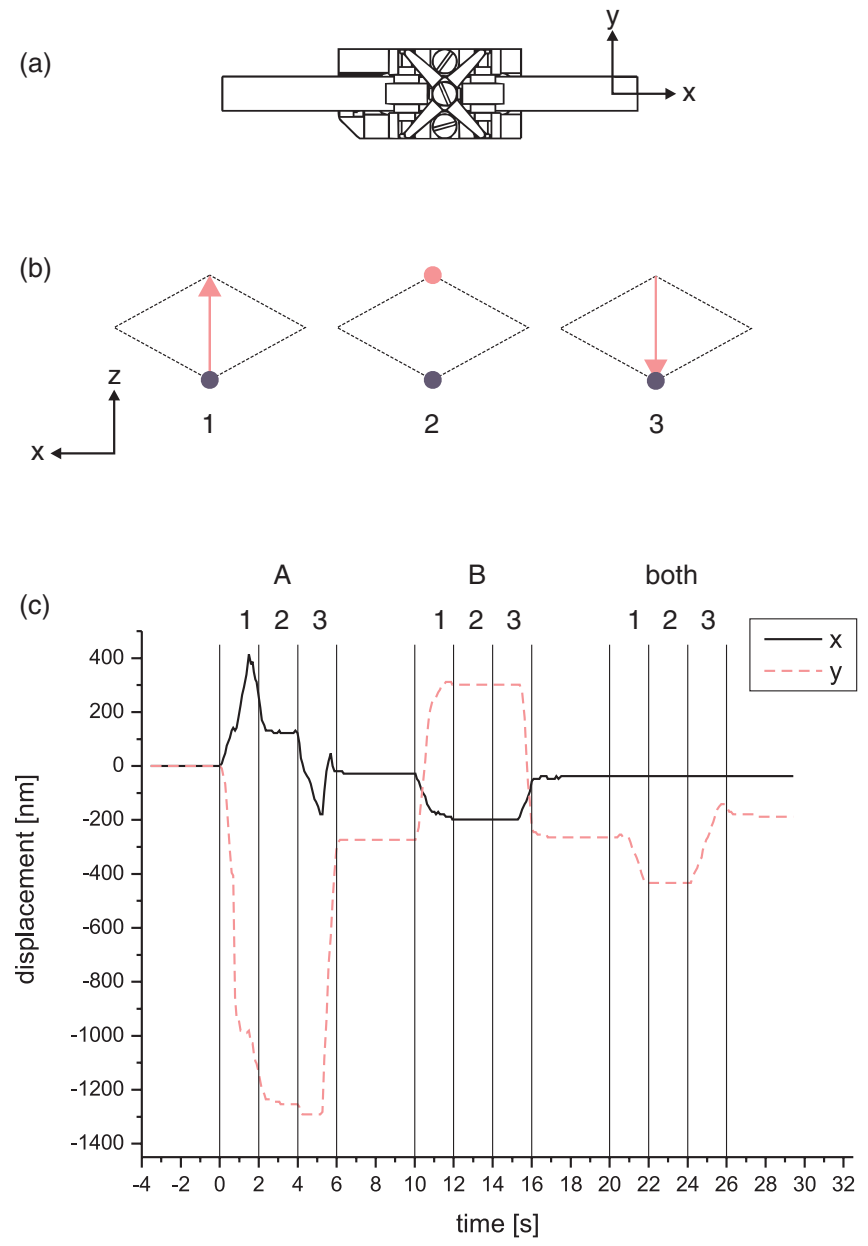

FIG. 5. Displacement of the bar applying $\mathrm{Z}$ displacement test signals: (a) schematic of the motor defining the directions. (b) Drive signals: (1) move up, (2) rest, (3) move down. (c) Displacements in X and Y direction of the bar resulting from the drive signal applied to pair A, pair B, and both pairs simultaneously. Note that we would expect no displacements for an ideal motor.

accuracy for displacement of this code is approximately one pixel, which amounts to a resolution of $9.5 \mathrm{~nm}$ for this measurement. However, there is an uncertainty in the total displacement of a few percent, due to adding up of the incremental uncertainties of the cross-correlation between images.

The displacement results are shown in Fig. 5(c) for the individual drive signals. In total, the bar displaces up to $415 \mathrm{~nm}$ in the positive X-direction, up to $198 \mathrm{~nm}$ in the negative $\mathrm{X}$-direction, up to $302 \mathrm{~nm}$ in the positive Y-direction, and up to $1.3 \mu \mathrm{m}$ in the negative Y-direction, while we had anticipated no movement at all. In the X-direction, the displacement is almost reversible, whereas in the Y-direction an offset is build up. The Y-displacement (measured at the end of the bar) may be caused by a linear displacement or a rotation of the bar: these two cases are undistinguishable due to the limited field of view.

Let us have a closer look at the $\mathrm{X}$-displacement, as this is the important axis of displacement of this motor. When pair A pushes up there is an overshoot to $415 \mathrm{~nm}$, before the bar comes to rest at $123 \mathrm{~nm}$, when pair A is in up position (phase 2). During the release of pair $A$, the bar overshoots to -179 $\mathrm{nm}$ and comes to rest at $-28 \mathrm{~nm}$, when pair $\mathrm{A}$ is back in 
the bottom position. It is striking that there are large overshoots during the upwards and downwards movement. The total displacement after operating pair A is $28 \mathrm{~nm}$. When operating pair $\mathrm{B}$, we do not find any overshoots. However, the $\mathrm{X}$-displacement moves from $-28 \mathrm{~nm}$ to $-198 \mathrm{~nm}$ and back to $-38 \mathrm{~nm}$ during operation. When operating both pairs, A and $\mathrm{B}$, simultaneously, we surprisingly do not find any measurable displacement in the X-direction. Please notice that an Xdisplacement of $\sim 320 \mathrm{~nm}=(123-(-198)) \mathrm{nm}$ is expected, if one pair completely takes over the bar from the other pair. ${ }^{65}$

We had expected no displacement of the bar at all, because the legs should only push in the Z-direction in this experiment. Apparently manufacturing tolerances in the legs (especially different lengths of the legs), alignment/mounting of the legs, roughness and/or thickness variations of the sliding bar, as well as inhomogeneities in the piezo material and different polarizations, cause a displacement in the X- and Y-plane, although the drive signal allows only for a pure Zmotion. Moreover, there might be a coupled X-Z displacement of the legs that, in addition, might differ from leg to leg. Surprisingly, Fig. 5(c) shows no X-displacement when both pairs move simultaneously. This might be a coincidence for this particular motor, such that the effect of pair A and pair $B$ just cancel each other. On the other hand, it might point towards a more general problem of these motors in relation to the forces on the individual roll bearings as explained in the following. As can be seen in Fig. 1 each pair is mounted asymmetrically with respect to the central axis of both roll bearings (see screw). This implies that if pair A moves up, the force on the left roll bearing is higher than the force on the right roll bearing. In turn, this leads to a larger Z-displacement of the left roll bearing with respect to the right one, and the associated torque might lead to a X-displacement of the bar towards the left. Symmetrical arguments hold for pair B. If the above is indeed the case, one should observe a general Xdisplacement of the bar in opposite directions, when pushing up pair A or pair B, respectively. This is exactly what we observe in Fig. 5(c). Also the direction of the displacement of the bar is correct: if pair A moves up, the bar is displaced in the positive $\mathrm{X}$-direction (note that the positive $\mathrm{X}$-direction is to the left in Fig. 1(b), whereas it is to the right in Fig. 5(a)). Moreover, when both pairs are pushed up simultaneously, the forces on both bearings are the same, there is no torque, and one should observe no displacement, which is again in perfect agreement with our observation.

The above implies that for the signals shown in Fig. 3, jumps (up to $415 \mathrm{~nm}$ ) and displacements (in the order of $320 \mathrm{~nm}$ ) will occur when one pair takes over the bar from the other piezo pair. An applied Z-displacement drive signal to one pair also introduces significant displacements of the bar in the $\mathrm{X}$ - and Y-direction.

\section{Problems of operation}

When operating the motor in fine mode, we observed jumps up to $2.4 \mu \mathrm{m}$. These jumps can be prevented, when the motor is operated more slowly such that the clamp (2), return (3), and release (4) phases are clearly separated in time and inertial forces are reduced. However, it is expected that in this mode intolerable overshoots occur that are as large as $415 \mathrm{~nm}$. Moreover, we also have a clear indication that an $\mathrm{X}$-displacement occurs, when one piezo pair takes over the bar from the other piezo pair. As a consequence, not only the fine mode is unsuitable but also the walking mode. As the taking over from one pair to the other in the walking mode with reduced step size (see Fig. 3(b)) can be compared to the clamping and releasing as sketched in Fig. 5(b), we expect that displacements of at least $\sim 320 \mathrm{~nm}$ will occur, next to the intolerable large overshoots. Finally, one might consider the walking mode operation as sketched in Fig. 3(a). We expect that this mode suffers from a friction problem. It is not clear at which precise height one pair takes over the bar from the other. As both pairs move in opposite directions, the local friction determines the final displacement of the bar. Moreover, strain can be built up in one piezo pair and released during the taking over leading to probably large jumps.

As it is impossible to produce an ideal motor, improved drive signals should take into account the problems arising from the manufacturing tolerances, the mounting/alignment of the legs and the bearings, the roughness and/or thickness variations of the sliding bar, the unideal movement of the piezo legs, the inhomogeneities in the piezo material, and different strengths of polarizations.

\section{Improved drive signal}

When developing improved drive signals the following two issues should be considered.

The first issue is the uncertainty in the precise moment that one pair takes over the bar from the other pair. Suppose pair $\mathrm{A}$ is at its maximum Z-range and pair $\mathrm{B}$ at its minimum Z-range, as depicted in phase 2 of Fig. 5(b). Then pair A holds the bar and pair $B$ is completely free of the bar. If we suppose now that pair A moves down and pair B moves up, the bar has to be taken over at some point by pair B from pair A. But the exact point of taking over does not necessarily coincide with the moment that the pairs are at equal Z-voltages. It is expected that this precise moment differs locally, as it depends on the long scale roughness of the bar, the manufacturing tolerances in size, and the piezoelectric coefficients of the legs. Furthermore, it is likely that the taking over does not occur at a certain point, but gradually over a range of Z-voltages. Finally, as described above, it is expected that a displacement occurs during the taking over.

The second issue is the strain in the legs, which arises when an external force pushes or pulls the bar, e.g., to the left. If pair A holds the bar, it is strained such that it exactly counteracts the external force. When pair B takes over the bar, it moves up and pair A moves down. This implies that the normal force on pair B increases, while the normal force on pair A decreases. The same holds for the friction force between the pairs and the bar and thus the leftward (external) force on pair B increases. As a consequence, the initially unstrained pair B will strain until the resulting stress counteracts the external force. This deformation causes the bar to displace in the direction of the external force. The magnitude of the 


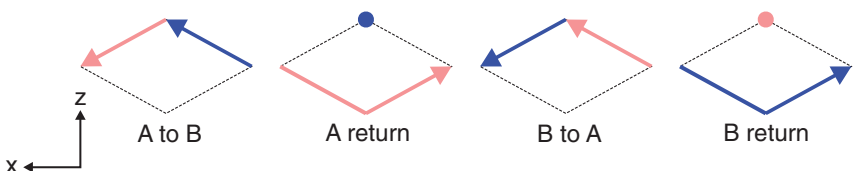

FIG. 6. Improved drive signal: light red represents pair A and blue pair B. (A to $B$ ) The legs move at the same $\mathrm{X}$-speed while they smoothly give over the bar from A to B. (A return) Leg B is extended to its maximum Z-position, thus holding the bar, while leg $\mathrm{A}$ is returning to its minimum $\mathrm{X}$ position. The full cycle of operation is completed by repeating these steps with interchanged pairs: (B to A) and (B return).

displacement depends on the stiffness of the legs and on the external force.

Considering the above formulated issues, we have developed the drive signal as depicted in Fig. 6. The main essence is that pair A gradually takes over from pair B while the legs move forward at approximately the same speed. There is neither a phase where the pairs move in different directions, like depicted in Fig. 3(a), nor is there a clamp-release phase, like depicted in Fig. 5(b). Moreover, the improved drive signal is insensitive to the exact position, or range, where taking over occurs, as long as it takes place during the "A to B" phase and not during the return phase. These features also circumvent the jumps and unwanted displacement due to strain in the legs. Finally, the displacement can be stopped (and reversed) at any time during the cycle, as the bar is continuously held by at least one pair operating in bending-mode.

\section{E. Application of improved drive signal}

Similar as described above we use the SEM to quantify the displacement of the bar in the X-direction, when the motor is operated with our improved drive signal. In addition, a spring is used to apply an external force of $0.34 \mathrm{~N}$ to the bar in the minus $\mathrm{X}$-direction. This is equivalent to three times the force that the sample holder will exert on the bar in our STM. The voltages lookup table used in our LabView program, which determines the resolution and the timing of the drive signal, consists of 1000 lines for the phases "A to B" and "B to A" and 2000 lines for the return phases. A break of 300 lines is inserted after the "A to B" phase to allow for a manual shift of the field of view of the SEM. In the experiment, we run the table at two different speeds: $500 \mathrm{~Hz}$ (fast) and $10 \mathrm{~Hz}$ (slow). A readjustment of the SEM followed by a recalibration revealed a resolution of $6.0 \mathrm{~nm} / \mathrm{pix}$.

Figures 7(a) and 7(b) show the displacement results for $500 \mathrm{~Hz}$ and $10 \mathrm{~Hz}$, respectively, in which the bar pushes against the force. The different phases in the cycles are indicated on top of the graphs.

Inspecting Fig. 7(a) one finds that there is a strong similarity between all phases "A to B" together with "A return" (called odd) as well as all phases "B to A" together with "B return" (called even). However, the odd and even displacements differ in size: odd $=3.1 \mu \mathrm{m}$ and even $=3.8 \mu \mathrm{m}$. This difference can be explained by a difference in piezoelectric constants, polarizations, or lengths of the legs between the pairs A and B. It is striking that each "B return" phase shows a bump (reversible overshoot) of approximately $0.15 \mu \mathrm{m}$, see (a)

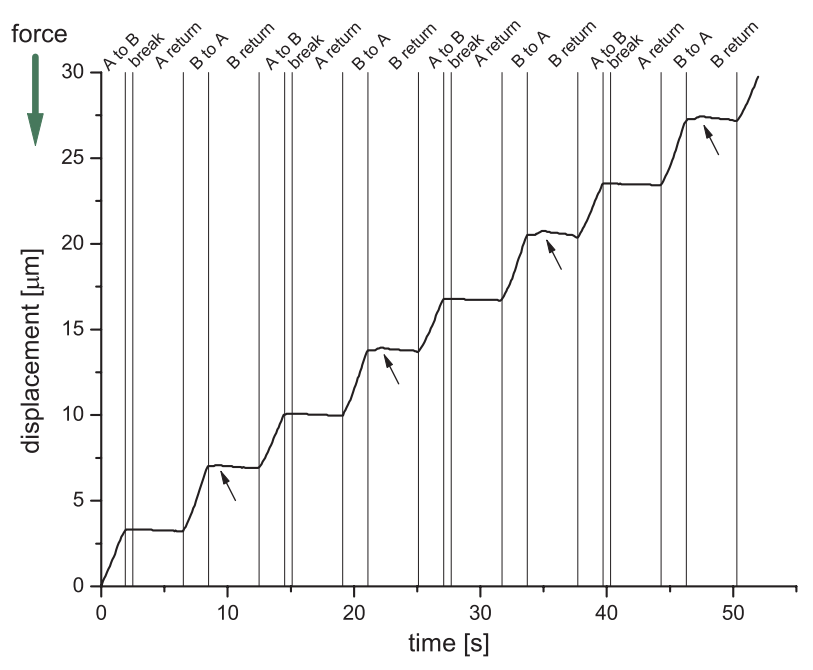

(b)

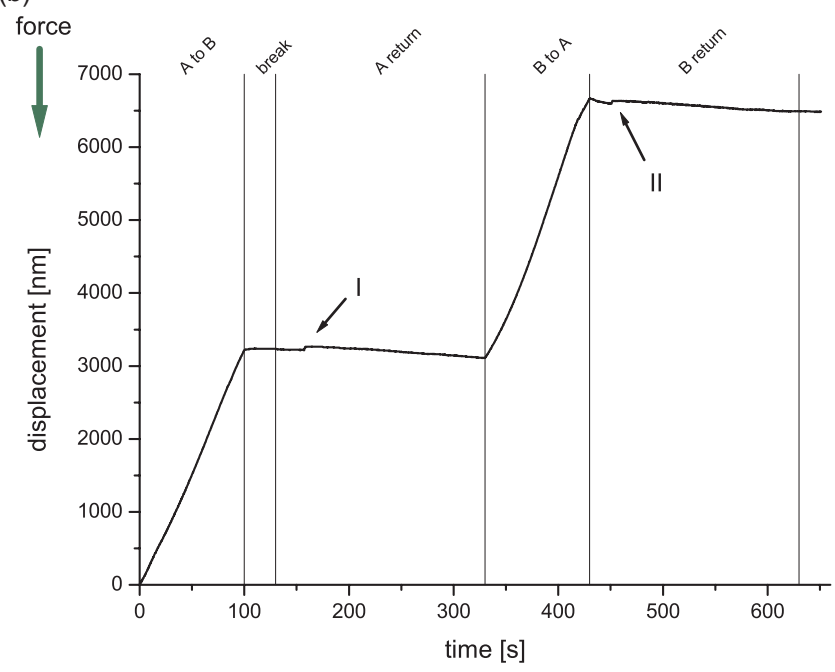

FIG. 7. Displacement of the bar applying improved drive signal: the bar is moving against an external force of $0.34 \mathrm{~N}$. (a) Fast movement $(500 \mathrm{~Hz})$; the arrows indicate reversible bumps of $\sim 0.15 \mu \mathrm{m}$ in the "B return" phase (see text). (b) Slow movement $(10 \mathrm{~Hz})$; I indicates an artifact of the SEM (see text) and II indicates a jump of $42 \mathrm{~nm}$, presumably based on the release of pair B (see text).

arrows in Fig. 7(a). These bumps are not classified as jumps, because the displacement before and after is equal. We do not have an explanation for these bumps, as they occur in the return phase, in which we expect the bar to be fully decoupled from pair B. The similarity within all even and all odd displacements indicates that the $\mathrm{nm}$-scale displacement is fully reproducible from cycle to cycle.

Figure 7(b) shows the displacement, when operating the motor in the slow mode $(10 \mathrm{~Hz})$. We see two discontinuities, labeled I and II. Feature I is an artifact of the measurement with the SEM: it corresponds to a switching of one of the lens currents thereby causing a shift of the image. Feature II indicates a jump of $42 \mathrm{~nm}$ at the start of the "B return" phase. The position of this jump coincides roughly with that of the bump, when operating the motor in the fast $(500 \mathrm{~Hz})$ mode. We do not see the above described bumps in the slow mode.

One explanation for the jump might be the following. Let us suppose that pair B is still in contact with the bar at the 
beginning of the "B return" phase, and that it pulls the bar with it during returning. Consequently, the displacement is slightly negative before the jump, as observed, and strain is built up in pair A. Since pair B also moves downward during returning, it loses contact with the bar at a certain moment. At this point, the strain of pair A is released leading to a jump of the bar in the forward direction, as indicated by II. This behavior can be explained by pair B being significantly taller than pair A. Other explanations might be based on the precise mounting of the legs, unideal movement of the legs, different piezoelectric coefficients, or surface inhomogeneities.

Finally, we observe a slight continuous backwards motion of the displacement during both "return" phases in Fig. 7(b). We attribute this to drift within the stage of the SEM, as we hardly see piezo creep of the motors in their final application, as explained in Secs. III F and III G.

As we have reduced the jumps from $2.4 \mu \mathrm{m}$ to less than $42 \mathrm{~nm}$ by applying our improved drive signal in slow mode operation, the motor is now suited to work as a coarse approach motor in an UHV-STM.

\section{F. Application as coarse approach motor}

We have designed and built the final microscope, ${ }^{60,61}$ in which we use three of these motors that hold a sample holder, as a coarse approach system (see Fig. 8). The microscope has been working reliably for more than 3 years and we never faced a problem with any of the motors. Although the motors have been tested to operate up to $70^{\circ} \mathrm{C}$, the UHV chamber (including the microscope with the motors) has been baked more than 30 times to $100{ }^{\circ} \mathrm{C}$ for $36 \mathrm{~h}$ reaching a base pressure of less than $5 \times 10^{-11}$ mbar. ${ }^{62}$ The motors are very stable. When operating the STM, we easily get atomic resolution and find that the drift in height (X-direction of the motors) at room temperature is approximately equal to the typical height drift

(a)

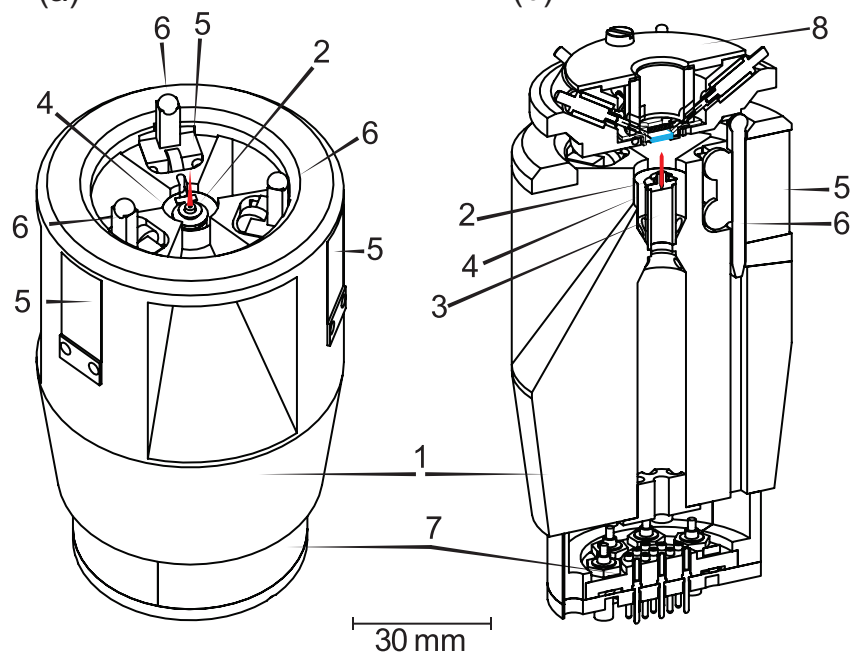

FIG. 8. Scanner body: (a) in full view without sample holder and (b) in crosssection placed with a sample holder on top. The components are: (1) body, (2) tip-holder and tip, (3) piezoelectric scanner, (4) shield to protect the piezoelectric scanner from the evaporation and sputtering sources, (5) approach motors (walking piezo motors), (6) moving bars of (5), (7) electrical connector, and (8) sample-holder. of a STM built without these motors, see, e.g., Ref. 63. Even at elevated temperatures of the sample the drift is very low, as the motors remain almost at room temperature: they are built within the main body of the microscope that acts as a heat sink. The rigidity of the motors is reflected in high resonance frequencies of the microscope and, therefore, its high-speed performance: we easily obtain video-rate imaging. ${ }^{4-47}$ It is to mention here that we had to apply a special DAC card (Measurement Computing PCI-DIO24H) to reach sufficiently high speed for setting the voltages as well as to build very lownoise piezo drivers for the motors. As the position of the motors and, therefore, also of the sample holder is determined by the voltage applied to the motors, the noise of the drivers determines the height stability. We designed drivers with a total output voltage range of $44 \mathrm{~V}$ and a noise below $10 \mu \mathrm{V}$ peakto-peak when loaded with the capacity of a leg. This ensures that the noise level corresponds to roughly $1 \%$ of the atomic corrugation of a fcc (111)-surface, which enables measurements with full atomic resolution in the STM.

\section{G. Calibrating the motors with the STM}

Using our new microscope $e^{60,61}$ in combination with our special SPM control electronics, ${ }^{45-47}$ which is also commercially available via, ${ }^{64}$ we have a (theoretical) height resolution of only $0.24 \mathrm{pm}$. If we do not reach this limit, the reason is our particular choice for a tunnel-current preamplifier with a large bandwidth or the existence of mechanical/acoustical noise, due to, e.g., still running turbomolecular pumps or a setup that is not mechanically isolated from its surroundings via air legs.

We have used this microscope to calibrate the step size of the motors by tunneling on a Si(100) surface. We control the motors with a home-built electronics that divides the full analog output range of $[-2 ; 42] \mathrm{V}$ over $2^{16}$ bits leading to a bit resolution of $0.67 \mathrm{mV} / \mathrm{bit}$. With the STM in tunneling conditions (but not scanning) and, therefore, under full height control via the feedback of the control electronics, we applied several series of digital steps to the different walking piezo motors and recorded with the highest possible resolution the change in height. One series consists of a repetition of 22 times applying quickly a certain number of DAC units. This leads to a stair case of height changes, as can be seen in Fig. 9(a) for 32 DAC units per step and Fig. 9(b) for 11 DAC units per step. The vibration in the height versus pixel/time data is due to two running turbomolecular pumps and a setup that was not floating on its air legs. From such graphs, we determined the mean step size and the standard deviation for a particular choice of DAC units per step and plotted the result in Fig. 10. One clearly observes a straight line that perfectly goes through zero. Also the standard deviation (see error bars) is decreasing with decreasing number of DAC units per step, which points towards a controllable and reliable motor that sets each individual step with the same precision. From the slope, we can determine the resolution of the motors, which is $27.4 \pm 0.6 \mathrm{pm} / D A C$ unit and therefore clearly in the low pm-range. This resolution is better and deviates significantly from the calibration obtained with the SEM (described in 

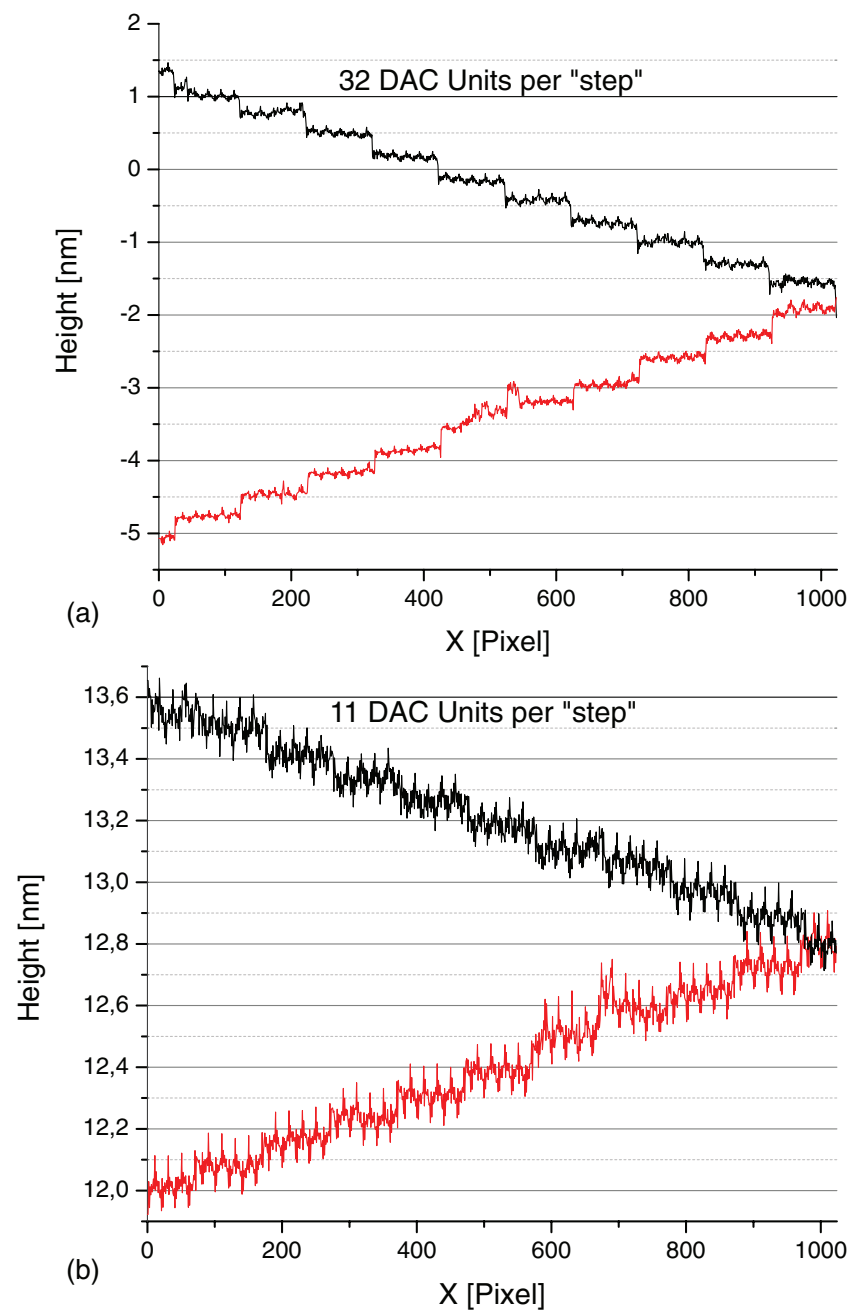

FIG. 9. Height changes measured in the STM under full feedback control for a series of digital steps applied to the walking piezo motors: one series consists of a repetition of 22 times applying quickly a certain number of DAC units. (a) shows the height changes for 32 DAC units per step, whereas (b) shows the height changes for 11 DAC units per step. The vibration in the height versus pixel/time data are due to two running turbomolecular pumps and a setup that was not floating on its air legs.

Sec. III A), but one should realize the limited resolution of the SEM and the extreme difference in accuracy between the STM and the SEM. We can conclude that the walking piezo motors can be controlled in the low pm-range and that the resolution of $27.4 \pm 0.6 \mathrm{pm} / D A C$ unit is, in principle, only limited by the bit depth of our home-built electronics for the walking piezo motors. In our design, the motors clearly exceed all requirements of approach motors of a vacuum SPM.

\section{H. Outlook: Further improvements}

Despite the successful implementation of the motors in the UHV-STM, there is still room for further improvement, if, e.g., needed:

For the particular motor tested, we have strong indications that one of the pairs (pair B) is still in contact with the bar during part of the return phase and that this leads to the $42 \mathrm{~nm}$ jumps. Removal of one or several of the springs of the motor reduces the normal force on the legs, which in turn reduces the compression of the legs. This results in a larger

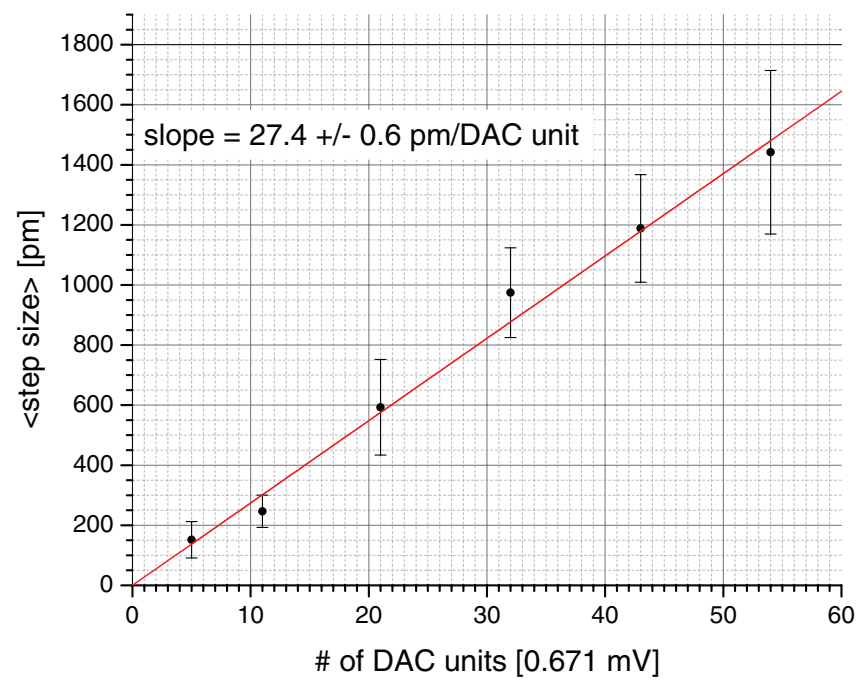

FIG. 10. Resolution of the walking piezo motor: From graphs shown in Fig. 9, we determined the mean step size and the standard deviation for a particular choice of DAC units per step. The slope represents the resolution of the motors, when controlled with our home-built electronics: the resolution is $27.4 \mathrm{pm} /$ DAC unit.

total Z-range of the legs and, therefore, in a reduced voltage difference to completely disconnect one pair. By tuning the normal force a situation should be achievable, in which the legs are surely disconnected from the bar during their return phase. However, this approach will have some consequences for the stability of the motor and, therefore, also for the stability of the tip-sample loop and the high-speed performance of the microscope.

A different approach would be to increase the Zdisplacement difference $(\Delta Z)$ during the return phase as shown in Fig. 11. A disadvantage, however, would be a reduced total $\mathrm{X}$-displacement $(\Delta X)$ and a slower forward speed during the taking over of the bar. In addition, the signal becomes more similar to that of the pure Z-displacement (see Fig. 5) and unwanted jumps and displacements might occur.

If the jumps and displacements are due to the different forces on the ball bearings and the related torques (see Sec. III B), a solution might be a different definition of the pairs of piezo legs. If one pair would consist of the two outer legs and the other pair of the two inner legs, the situation regarding the forces on the bearings would be completely

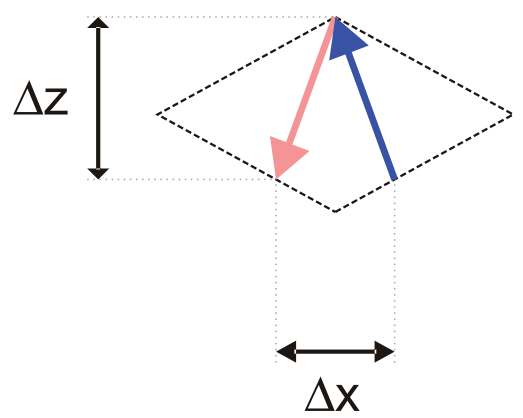

FIG. 11. Suggested additionally improved drive signal: increasing $(\Delta Z)$ for the "A to B" and "B to A" phase will increase the likelihood that the returning pair is not in contact with the bar during its "return" phase. 
symmetric. Torque terms as well as involved displacements should be absent in this case.

\section{CONCLUSION}

We have investigated and improved the performance of a walking piezo motor. When applying the standard drive signals, we find large jumps, up to $2.4 \mu \mathrm{m}$, in the $\mathrm{X}$ displacement. We analyzed possible mechanisms for these jumps and tested the motor performance with respect to pure Z-displacement. Again we found large displacements in the $\mathrm{X}$-direction, whereas for an ideal motor one would expect no movement at all. Unfortunately, the production of an ideal motor is impossible, as one has to deal with manufacturing tolerances, the mounting/alignment of the legs, the roughness and/or thickness variations of the sliding bar, a coupled X-Z movement of the piezo legs, the inhomogeneities in the piezo material, and different polarizations. Nevertheless, we have solved this problem by developing an improved drive signal. The essential feature of this signal is that the bar is gradually taken over from one pair to the other while both pairs move forward at approximately the same speed. When applying our improved signal, the jumps are reduced to less than $42 \mathrm{~nm}$. This jump size is sufficiently low for our application and we successfully implemented these motors as a coarse approach system in a video-rate UHV-STM. Using this STM, we could even show that it is possible to reach a resolution of $27 \mathrm{pm}$ with the walking piezo motors. Finally, in an outlook we provided possibilities for further improvements, if the jump size is still too large for a specific application.

We expect that the general behavior will hold for all motors that are based on a similar principle: the shared problem is the taking over (of a slider or a fixed wall) when the piezo element(s) reach(es) the end of its (their) range. The solutions presented in this paper should, therefore, also improve the displacement accuracy of any of these motors.

\section{ACKNOWLEDGMENTS}

We kindly acknowledge J. W. M. Frenken for his comments on friction and F. Galli and P. C. van der Tuijn for proofreading of this paper.

${ }^{1}$ B. N. J. Persson, Sliding Friction: Physical Principles and Applications (Springer, Berlin, 1998).

${ }^{2}$ J. Krim, Phys. World 18, 31 (2005).

${ }^{3}$ S. Yu. Krylov and J. W. M. Frenken, Phys. Status Solidi (b) 251(4), 711-736 (2014).

${ }^{4}$ B. J. Albers, M. Liebmann, T. C. Schwendemann, M. Z. Baykara, M. Heyde, M. Salmeron, E. I. Altman, and U. D. Schwarz, Rev. Sci. Instrum. 79, 033704 (2008)

${ }^{5}$ Chr. Wittneven, R. Dombrowski, S. H. Pan, and R. Wiesendanger, Rev. Sci. Instrum. 68, 3806 (1997).

${ }^{6}$ S. H. Pan, E. W. Hudson, and J. C. Davis, Rev. Sci. Instrum. 70, 1459 (1999).

${ }^{7}$ S. H. Pan, International Patent Publication No. WO 93/19494 (30 September 1993).

${ }^{8}$ A. K. Gupta and K.-W. Ng, Rev. Sci. Instrum. 72, 3552 (2001).

${ }^{9}$ B. Drevniok, W. M. P. Paul, K. R. Hairsine, and A. B. McLeanb, Rev. Sci. Instrum. 83, 033706 (2012).

${ }^{10}$ R. A. Wolkow, Rev. Sci. Instrum. 63, 4049 (1992)

${ }^{11}$ Burleigh Instruments, Inc., U.S. patent 3,902,084 (26 August 1975).

${ }^{12}$ S. Hsu, A. Arbor, and A. Blatter, U.S. patent 3,292,019 (1 November 1963).
${ }^{13}$ G. R. Stibitz, U.S. patent 3,138,749 (5 March 1962).

${ }^{14}$ C. G. O'Neill and C. E. Foster, U.S. patent 4,219,755 (18 March 1977).

${ }^{15}$ G. V. Galutva, U.S. patent 3,684,904 (15 April 1970).

${ }^{16} \mathrm{~T}$. Fujimoto, U.S. patent 4,736,131 (30 July 1986).

${ }^{17}$ P. E. Tenzer and R. Ben Mrad, IEEE/ASME Trans. Mechatron. 9, 427 (2004).

${ }^{18}$ J. Frank, G. H. Koopmann, W. Chen, and G. A. Lesieutre, Proc. SPIE 3668, 717 (1999).

${ }^{19} \mathrm{~J}$. Ni and Z. Zhu, IEEE/ASME Trans. Mechatron. 5, 441 (2000).

${ }^{20}$ K. Duong and E. Garcia, Proc. SPIE 2443, 782 (1995).

${ }^{21}$ J. E. Miesner and J. P. Teter, Proc. SPIE 2190, 520 (1994).

${ }^{22}$ K. Besocke, Surf. Sci. 181, 145 (1987).

${ }^{23}$ T. H. Chang, C. H. Yang, M. J. Yang, and J. B. Dottellis, Rev. Sci. Instrum. 72, 2989 (2001)

${ }^{24}$ J. H. Ferris, J. G. Kushmerick, J. A. Johnson, M. G. Y. Youngquist, R. B. Kessinger, H. F. Kingsbury, and P. S. Weisse, Rev. Sci. Instrum. 69, 2691 (1998).

${ }^{25}$ N. Pertaya, K.-F. Braun, and K.-H. Rieder, Rev. Sci. Instrum. 75, 2608 (2004).

${ }^{26}$ L. A. Silva, Rev. Sci. Instrum. 68, 1300 (1997).

${ }^{27}$ B. Koc, S. Cagatay, and K. Uchino, IEEE Trans. Ultrason. Ferroelectr. Freq. Control 49, 495 (2002).

${ }^{28} \mathrm{M}$. Bexell and S. Johansson, Sens. Actuators A 75, 118 (1999).

${ }^{29}$ J. Frohn, J. F. Wolf, K. Besocke, and M. Teske, Rev. Sci. Instrum. 60, 1200 (1989).

${ }^{30}$ Th. Michely, M. Kaiser, and M. J. Rost, Rev. Sci. Instrum. 71, 4461 (2000).

${ }^{31}$ Th. Michely, M. J. Rost, and M. Kaiser, U.S. patent 6,603,239 B1 (11 July 2000).

${ }^{32}$ D. W. Pohl, Rev. Sci. Instrum. 58, 54 (1987).

${ }^{33}$ Y. Hou, J. Wang, and Q. Lu, Rev. Sci. Instrum. 79, 113707 (2008).

${ }^{34}$ R. Yoshida, Y. Okamoto, and H. Okada, J. Jpn. Soc. Precis. Eng. 68, 536 (2002).

${ }^{35}$ W. Zesch, R. Buchi, A. Codourey, and R. Siegwart, Proc. SPIE 2593, 80 (1995).

${ }^{36}$ D.-S. Paik, K.-H. Yoo, C.-Y. Kang, B.-H. Cho, S. Nam, and S.-J. Yoon, J. Electroceram. 22, 346 (2009).

${ }^{37}$ L. Howald, H. Rudin, and H.-J. Güntherodt, Rev. Sci. Instrum. 63, 3909 (1992).

${ }^{38}$ J. W. Judy, D. L. Polla, and W. P. Robbins, IEEE Trans. Ultrason. Ferroelectr. Freq. Control 37, 428 (1990).

${ }^{39}$ M. J. Brukman and R. W. Carpick, Rev. Sci. Instrum. 77, 033706 (2006).

${ }^{40}$ S. Behler, M. K. Rose, D. F. Ogletree, and M. Salmeron, Rev. Sci. Instrum. 68, 124 (1997).

${ }^{41}$ Q. Wang and Q. Lu, Rev. Sci. Instrum. 80, 085104 (2009).

${ }^{42}$ D. Kang, M. G. Lee, and D. Gweon, Rev. Sci. Instrum. 78, 075112 (2007).

${ }^{43}$ X. Liu and Q. Lu, Rev. Sci. Instrum. 83, 115111 (2012).

${ }^{44}$ V. Cherepanov, P. Coenen, and B. Voigtländer, Rev. Sci. Instrum. 83, 023703 (2012).

${ }^{45}$ M. J. Rost, L. Crama, P. Schakel, E. van Tol, G. B. E. M. van VelzenWilliams, C. F. Overgauw, H. ter Horst, H. Dekker, B. Okhuijsen, M. Seynen, A. Vijftigschild, P. Han, A. J. Katan, K. Schoots, R. Schumm, W. van Loo, T. H. Oosterkamp, and J. W. M. Frenken, Rev. Sci. Instrum. 76, 053710 (2005).

${ }^{46}$ G. Schitter and M. J. Rost, Mater. Today 11, 40 (2008).

${ }^{47}$ M. J. Rost, G. J. C. van Baarle, W. M. van Spengen, P. Schakel, W. A. van Loo, A. J. Katan, T. H. Oosterkamp, and J. W. M. Frenken, Asian J. Control 11(2), 110 (2009).

${ }^{48}$ Y. I. Yanson, F. Schenkel, and M. J. Rost, Rev. Sci. Instrum. 84, 023702 (2013).

${ }^{49}$ G. C. Dong, D. W. van Baarle, M. J. Rost, and J. W. M. Frenken, New J. Phys. 14, 053033 (2012).

${ }^{50}$ M. J. Rost, Phys. Rev. Lett. 99, 266101 (2007).

${ }^{51}$ M. J. Rost, D. A. Quist, and J. W. M. Frenken, Phys. Rev. Lett. 91, 026101 (2003).

${ }^{52}$ Y. I. Yanson and M. J. Rost, Angew. Chem., Int. Ed. 52, 2454 (2013).

${ }^{53}$ V. Fokkema, J. Verhoeven, J. W. M. Frenken, and M. J. Rost, "Direct observation of ion erosion," Nat. Mater. (submitted).

${ }^{54}$ R. J. E. Merry, M. G. J. M. Maassen, M. J. G. van de Molengraft, N. van de Wouw, and M. Steinbuch, IEEE Trans. Mech. 16, 615 (2011).

${ }^{55}$ R. Merry, R. van de Molengraft, and M. Steinbuch, Sens. Actuators A 162, 51 (2010).

${ }^{56}$ Micromo, see http://www.micromo.com.

${ }^{57} \mathrm{Physik}$ Instrumente (PI) GmbH, see http://www.physikinstrumente.com. 
${ }^{58}$ PiezoMotor, see http://www.piezomotor.se.

${ }^{59}$ Piezo Technology Tutorial PI Ceramic; see http://www.piceramic.com/ pdf/Piezoelectric_Effect_Piezo_Techlology_Tutorial_PI_Ceramic.pdf.

${ }^{60}$ M. den Heijer, V. Fokkema, G. Verdoes, A. C. Geluk, G. Schitter, and M. J. Rost, "Towards a faster scanning tunneling microscope with finite element analysis and vibrometer measurements," Rev. Sci. Instrum. (submitted).

${ }^{61}$ V. Fokkema, G. Verdoes, A. C. Geluk, J. Verhoeven, and M. J. Rost, "A video-rate scanning tunneling microscope for real-time observation of deposition and ion bombardment," Rev. Sci. Instrum. (submitted).

${ }^{62} \mathrm{We}$ applied a special surface treatment to the chamber such that UHV can be achieved when baking the chamber only to $100{ }^{\circ} \mathrm{C}$ : the chamber was vacuum fired for several hours at $\sim 900^{\circ} \mathrm{C}$ at $V G$ Scienta.
${ }^{63}$ M. S. Hoogeman, D. G. van Loon, R. W. M. Loos, H. G. Ficke, E. de Haas, J. J. van der Linden, H. Zeijlemaker, L. Kuipers, M. F. Chang, M. A. J. Klik, and J. W. M. Frenken, Rev. Sci. Instrum. 69, 2072 (1998).

${ }^{64}$ Leiden Probe Microscopy, see http://www.leidenprobemicroscopy.com.

${ }^{65}$ Strictly speaking this holds only, if one compares the situations before and after with completely extended pairs. There is evidence in Fig. 5(c) that the total X-displacement might linearly depend on the applied Z-voltage. This would imply that the jump size is reduced, if the bar is taken over from one pair to the other at "half" of the total Z-voltage, like, e.g., shown in Fig. 3(a). In this case, one would expect a total X-displacement of $0.5 \times 123$ $\mathrm{nm}-0.5 \times(-198) \mathrm{nm}=160 \mathrm{~nm}$. 